Wolfgang Niederlag, Heinz U. Lemke, Hans Lehrach, Heinz-Otto Peitgen Der virtuelle Patient 


\section{Health Academy}

Herausgegeben von

Wolfgang Niederlag und Heinz U. Lemke

\section{Band 1}


Wolfgang Niederlag, Heinz U. Lemke, Hans Lehrach, Heinz-Otto Peitgen

\section{Der virtuelle Patient}

2., erweiterte Auflage

\section{DE GRUYTER}


Herausgeber

Prof. Dr. rer. nat. Wolfgang Niederlag

Krankenhaus Dresden-Friedrichstadt

Friedrichstraße 41

01067 Dresden, Germany

Prof. Dr.-Ing. Heinz U. Lemke

University of Southern California

Los Angeles (USA) und IFCARS Office

Im Gut 15

79790 Küssaberg, Germany
Prof. Dr. rer. nat. Hans Lehrach

Max-Planck-Institut für Molekulare Genetik

Ihnestraße 63-73

14195 Berlin, Germany

Prof. Dr. rer. nat. Heinz-Otto Peitgen

Fraunhofer-Institut für Bildgestützte

Medizin MEVIS

Universitätsallee 29

28359 Bremen, Germany

1. Auflage 2012, (C) Health Academy, Dresden 2012

ISSN 1617-8874, ISBN 978-3-00-040331-6

Das Buch enthält 155 Abbildungen und 7 Tabellen.

ISBN: 978-3-11-033429-6

e-ISBN: 978-3-11-033566-8

ISSN: $2199-2959$

\section{Library of Congress Cataloging-in-Publication data}

A CIP catalog record for this book has been applied for at the Library of Congress.

\section{Bibliografische Information der Deutschen Nationalbibliothek}

Die Deutsche Nationalbibliothek verzeichnet diese Publikation in der Deutschen Nationalbibliographie; detaillierte bibliografische Daten sind im Internet über http://dnb.dnb.de abrufbar.

Der Verlag hat für die Wiedergabe aller in diesem Buch enthaltenen Informationen mit den Autoren große Mühe darauf verwandt, diese Angaben genau entsprechend dem Wissensstand bei Fertigstellung des Werkes abzudrucken. Trotz sorgfältiger Manuskriptherstellung und Korrektur des Satzes können Fehler nicht ganz ausgeschlossen werden. Autoren und Verlag übernehmen infolgedessen keine Verantwortung und keine daraus folgende oder sonstige Haftung, die auf irgendeine Art aus der Benutzung der in dem Werk enthaltenen Informationen oder Teilen davon entsteht.

Die Wiedergabe der Gebrauchsnamen, Handelsnamen, Warenbezeichnungen und dergleichen in diesem Buch berechtigt nicht zu der Annahme, dass solche Namen ohne weiteres von jedermann benutzt werden dürfen. Vielmehr handelt es sich häufig um gesetzlich geschützte, eingetragene Warenzeichen, auch wenn sie nicht eigens als solche gekennzeichnet sind.

(C) 2014 Walter de Gruyter GmbH, Berlin/Boston

Satz: Werksatz Schmidt \& Schulz GmbH, Gräfenhainichen

ArtConText (Berlin), Christine Knauber

Druck und Bindung: $\mathrm{CPI}$ books $\mathrm{GmbH}$, Leck

Einbandabbildung: Tom Barrick, Chris Clark, sghms/science photo library

(2) Gedruckt auf säurefreiem Papier

Printed in Germany

www.degruyter.com 